\title{
Physiotherapy clinical guidelines for Huntington's disease
}

\author{
Lori Quinn \& Monica Busse; On behalf of the European Huntington's \\ Disease Network Physiotherapy Working Group
}

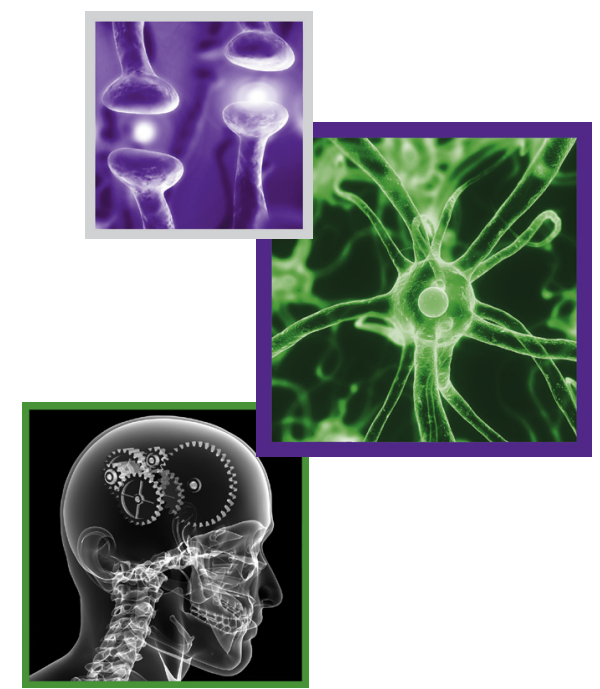

\section{Clinical implementation strategy}

People with Huntington's disease (HD) demonstrate a range of physical, cognitive, psychological and social care needs over an extended time frame. One of the difficulties in developing clinical guidelines for complex neurodegenerative diseases, such as HD, is the heterogeneity of clinical signs and symptoms. While staging of the disease process (e.g., early, middle, late) can provide a general framework for intervention, within each stage there is a wide range of potential impairments that can impact an individual's level of functional activity and life participation. This makes structuring of consistent therapeutic approaches problematic. This problem is not unique to HD, and has been documented in other physiotherapy patient groups, most notably low back and neck dysfunction, and for general neurorehabilitation patient groups [1-3].

Clinical guidelines are evidenced-based recommendations for clinical practice in specific conditions $[4,5]$. The availability of clinical guidelines facilitates uniformity of care and standards of practice with the aim of improving quality of care provision. It is critically important for health professionals to be able to define and document their assessment and intervention strategies. The application of appropriate guidelines in practice, as well as systematic outcome evaluation, has the potential to promote evidenced-based delivery of care for the benefit of the person with HD.

Members of the Physiotherapy Working Group (PWG) are advocating a treatment-based classification approach to specifically guide intervention strategies in HD. It is hoped that by creating subclassifications of patients' impairments and problems, and matching these with more specific interventions, therapists may improve outcomes in their patients. The seven classifications, presented in this report are listed and described in Table 1 below. These have been developed to better categorize patients with HD, who can present with a wide array of functional problems and physical, cognitive and behavioral impairments. It is the aim of the PWG that these treatment-based classifications will subsequently inform evaluation of complex interventions and advance research into care and evidenced-based service delivery for people with HD. As the complex nature of HD makes it unlikely that any one professional will have all the skills needed for best practice, these classifications have also incorporated referral to relevant members of the multidisciplinary team

Therapists should review the signs and symptoms of each classification to determine the best fit for a particular patient. Once a classification has been determined, therapists should use it as a guide to selecting appropriate evaluation measures and intervention strategies. Importantly, these classifications are not designed to take the place of independent clinical decision-making based on each patient's particular signs and symptoms, but rather should provide a framework for more consistent patient management through the disease spectrum. These classifications now require further clinical validation and will be reviewed periodically. were developed by a subgroup of physiotherapists: Lori Quinn, Monica Busse, Hanan Khalil, Una Jones, Angela Hall, Sue Armstrong. Subsequent development and field testing completed by members of the EHDN Physiotherapy Working Group, with specific contributions by: Karin Bunnig, Maggie Broad, Katy DeBono, Camilla Ekwall, Hanne Fossmo, Nora Fritz, Karen Jones, Una Jones, Deb Kegelmeyer, Hanan Khalil, Anne Kloos, Rodolfo Vera, Alexandra Schuler and Jessie van der Bent. 
SPECIAL REPORT Quinn \& Busse

\section{Table 1. Classifications for physiotherapy patient management.}

\section{Classification}

A. Exercise capacity and performance

B. Planning and sequencing of tasks (including bradykinesia)

C. Mobility, balance and falls risk

D. Secondary adaptive changes and deconditioning

E. Impaired postural control and alignment in sitting

F. Respiratory dysfunction

G. End stage care

\section{Description}

Absence of motor impairment or specific limitations in functional activities; potential for cognitive and/or behavioral issues

Presence of apraxia or impaired motor planning; slowness of movement and/or altered force generation capacity resulting in difficulty and slowness in performing functional activities

Ambulatory for community and/or household distances; impairments in balance, strength or fatigue resulting in mobility limitations and increased falls risk

Musculoskeletal and/or respiratory changes resulting in physical deconditioning, and subsequent decreased participation in daily living activities, or social/work environments

Altered alignment due to adaptive changes, involuntary movement, muscle weakness and incoordination resulting in limitations in functional activities in sitting

Impaired respiratory function and capacity; limited endurance; impaired airway clearance resulting in restrictions in functional activities and risk for infection

Active and passive range of motion limitations and poor active movement control resulting in inability to ambulate; dependent for most activities of daily living; difficulty maintaining upright sitting position

\section{Stages}

Pre-manifest/early

Early-mid

Early-mid

Early-mid

Mid-late

Mid-late

Late 
A. Exercise capacity and performance.

Description: absence of motor impairment or specific limitations in functional activities; potential for cognitive and/or behavioral issues; stage: pre-manifest/early.

Signs and General aims Treatment options

symptoms/

key issues and

potential

issues

Participation: - Health education and advice including

No problems.

Activities:

No problems.

Impairments:

Absence

of motor

impairment.

Potential for:

cognitive and/

or behavioral

issues; poor

endurance and

limited physical

activity; lack

of motivation

and/or apathy;

anxiety and/

or depression

$[6,7]$; sleep

disturbance,

which may

exacerbate

the above

impairments. general health promotion strategies, referral for exercise on prescription, and agenda setting to optimize exercise performance.

- Patient \& family education on the importance of early intervention in $\mathrm{HD}^{+}$.

- Baseline testing for fitness level should be completed prior to exercise prescription. Consider education on fatigue and the timing of intervention/ exercises during the day as well as careful instruction on safety during exercise.

- Identify barriers and facilitators to initiate and maintain an exercise program [13]. Help patient identify barriers and facilitators and explore strategies to manage them [14]. Involvement of a caregiver/friend/ spouse can make the program more successful.
Gym-based exercises can be of benefit to physicallyable individuals, as well as people
with long-term neurological conditions, such as Parkinson's disease [15]. Small case
reports in HD [16,17] indicate that if properly supported, people with HD can enjoy
the health benefits of physical activity. Physiotherapists report that early referral
to establish relationships and promote sustainable exercise behavior would be
preferred [18].

- Individualized goal setting and home exercise program prescription for optimization of services in a life-long disease process.

- A focus on task-specific functional activities incorporated into the exercise program.

- A warm-up and cool-down.

- Careful monitoring of vital signs, dyspnea, fatigue, pallor, dizziness and specific HD-related signs at rest, during and after exercise.

- Frequency, intensity, duration and mode are dependent on the baseline fitness level of the individual; however, focus should be on exercise for both aerobic and strength training [19].

\section{Aerobic exercise:}

- Frequency: 3-5-times a week; intensity: 65-85\% of the maximal heart rate; 55-65\% of maximal heart rate for deconditioned individuals; Duration: at least 30 min of continuous or intermittent training per day (minimum of 10-min bouts accumulated throughout the day); Mode: any activity that the individual enjoys that uses large muscle groups which can be maintained continuously and is rhythmic and aerobic in nature (e.g., walking, jogging, swimming, and biking).

Resistance exercises:

- Frequency and duration: 8-12 repetitions per exercise; one set of 8-10 exercises that conditions large muscle groups 2-3-times a week; Intensity: $65-70 \%$ of 1 rep max for upper body and $75-80 \%$ of 1 rep max for lower body parts; Mode: Resistance training should be progressive and individualized.

- Exercise program ideas: walking (treadmill and over-ground), stationary bicycling, horse-back riding, strength-training, balance training, core stability training, videogame based exercise (Nintendo Wii, Dance Dance Revolution) [20]. Yoga, pilates, tai chi and relaxation are also recommended.

\section{Outcome measures:}

PAR-Q: The Physical Activity Readiness Questionnaire can be utilized as an initial screening method to determine any contraindications to exercise. If any reason to doubt safety in participating in exercise, the physiotherapist should refer persons to their GPs for a full assessment [21].

IPAQ: International Physical Activity Questionnaire: is a useful questionnaire for obtaining information about weekly activity levels at home, work and leisure. It is reliable and valid in 18-65-year-old healthy adults as an epidemiological measure. It is not valid as a measure of change or to assess the effects of an intervention [22].

Activity monitor: if available, a useful measure of physical decline in HD as hypokinesia is related to functional capacity in HD. May also be used to show change in activity over time [23-26].

Borg RPE (or CR-10): is a useful objective measure of perceived exertion. In addition, it allows patients to self-monitor during their exercise routine. This RPE (rate of perceived exertion) scale has become a standard method for evaluating perceived exertion in exercise testing, training and rehabilitation, and has been validated against objective markers of exercise intensity [27,28].

Goal attainment scale: assists with appropriate goal setting, quantifying achievement, and even weighting of goals to reflect the personal importance of the goal to the patient and/or the opinion of the therapist on the difficulty of achieving the goal [29].

6-min walk test (BMWT): for measuring endurance and cardiovascular fitness [30].

\section{Interdisciplinary:}

Nutrition: assessment and management of caloric intake, dietary needs and changes to diet when exercising.

Genetic counselling/psychology: assessment and management of behavioral issues.

Occupational therapy: assessment and management of activity of daily living-related deficits.

Speech \& language therapy: for clear communication of printed exercise routines.

${ }^{+}$In animal models of HD, early enrichment of the environment (i.e., exercise) was shown to delay symptom onset and severity [8]. Increased physical activity was critical for successful outcomes of disease modifying treatments such as cell transplants, reconnection of grafted tissue and dopamine innervations [9]. Active lifestyle is also thought to delay the disease onset in people with HD [10] and to reduce the risk of other neurodegenerative disease, such as Alzheimer's disease [11] and Parkinson's disease [12]. In addition, participation in regular exercise has potential to result in improved strength, cardiovascular fitness, exercise tolerance, functionality, mobility and mood. 


\section{B. Planning and sequencing of tasks.}

Description: presence of apraxia or impaired motor planning; slowness of movement and/or altered force generation capacity resulting in difficulty and slowness in performing functional activities; Stage: early-mid.

Signs and symptoms/key issues and potential issues

\section{Participation:}

Patients may note a decline in independence in their daily routine, and may experience difficulty managing previously automatic tasks, such as rising from a chair or walking.

Activities:

Difficulty and slowness in performing functional activities (dressing, bathing, stair climbing, indoor/outdoor ambulation, ADLs).

\section{Impairments:}

Apraxia - impaired motor planning [31]; movement speed - slowed speed of movement during purposeful task; force generation generalized and/or specific muscle weakness; delayed onset of muscle response; safety awareness and insight into deficits.
General aims

- Creation of individualized patient-centered goals that focus on the specific impairments of the patient

Examples include:

- Improve ability to perform functional tasks

- Increase speed of movement

- Maximize safety
Treatment options

Task-specific training to address planning and sequencing deficits. Could include:

- Strategy training in daily living activities: this technique teaches internal (for example, the patient is taught to verbalise and implement the task steps at the same time) or external (for example, when aids are used to overcome a functional barrier) compensatory strategies that enable a functional task to be completed [32]; spaced retrieval and errorless learning techniques can guide training of motor activities and learning of skilful activities [33].

- Sensory stimulation: deep pressure and soft touch are applied to the patients' limbs; therapies utilizing a multi-sensory stimulation approach noted significant improvements in mood and stimulation which were cumulative over sessions when compared with the control group [34].

- Cueing: visual, verbal or physical prompts: verbal prompts can include attentional strategies with external cues and/or, attentional strategies with internal cues.

- Chaining (forward or backward): the task is broken down into its component parts. Using backward chaining the task is completed with facilitation from the therapist apart from the final component, which the patient carries out unaided. If successful, further steps are introduced in subsequent trials.

\section{Outcome measures:}

SF-36: A quickly and easily administered Quality of Life measure commonly used in HD; robust construct validity and test-retest reliability have been demonstrated [35].

10-m walk: used as a measure of gait speed [36].

Physical Performance Test (PPT): useful for measuring ability to perform everyday tasks including utensil use, writing, donning/doffing a jacket, walking and stair climbing.

Goal attainment scale: see $A$.

Timed Up \& Go (TUG): The participant is instructed to stand from a chair, walk 10 feet, return to the chair and be seated. Times greater than 13.5 seconds accurately predicted elderly fallers with $90 \%$ accuracy. The TUG has been validated in HD [26,37].

TUG Manual complete TUG while carrying a full cup of water; a time difference of $>4.5$ seconds between TUG and TUG Manual indicated increased risk of future falls in elderly.

TUG Cognitive (gait speed; dual-task ability) complete the TUG while counting backwards from a randomly selected number between 20 and 100 [38].

Four Square Step Test (4SST): participants step forward, sideways and backward over low obstacles (usually canes) in a sequence; a useful measure of balance, stepping ability and motor planning [39].

The Apraxia Test: consists of two subtests assessing the ability to use objects or pantomime use and the ability to imitate gestures [40].

Timed Sit-to-Stand Test: patients are asked to stand up and sit back down five-times from a chair that reaches the head of their fibula. Time to complete 5 repetitions are recorded. The Sit-to-Stand test is commonly used to assess leg strength and balance and has been shown to be a reliable and valid measure in older adults and other patient populations [41].

\section{Interdisciplinary:}

Occupational therapy: problem solving and strategies for successful completion of activities of daily living. Compensatory strategies for managing deficits in ocular movements [42] and early cognitive impairments.

Speech and Language Therapy: assessment and management of communication (dysarthria and apraxia of speech; influence of cognitive ability in particular difficulties in executive functioning and in working memory on linguistics, speech and communication skills); assessment and management of swallowing and dysphagia and any association with motor function/ planning problems. 
C. Mobility, balance and falls risk.

Description: ambulatory for community and/or household distances; impairments in balance, strength or fatigue resulting in mobility limitations and increased falls risk; stage: early-mid.

Signs and symptoms/ key issues and potential issues

\section{Participation:}

Fear of falling may result in more unwillingness to participate in home, work, and community activities; Difficulty in participating in recreational sports (e.g., cycling, running, soccer, basketball) that require balance and mobility.

\section{Activities:}

Difficulty walking in certain environments (i.e., open environments); Difficulty walking backwards or sideways; Difficulty turning and changing directions; Difficulty getting in and out of chairs and beds due to vaulting, poor eccentric control; Difficulty with walking while doing a secondary cognitive task due to attentional deficits [43]; High falls risk [26,44].

Impairments:

- Bradykinesia [45]; Dystonia - affecting trunk (lateral shift; extension), ankles/feet (inversion) [46]; Chorea ${ }^{\dagger} /$ rigidity [47-49]; Muscle weakness or impaired force production [50]; Impaired motor control (i.e., force modulation deficits causing sudden exaggerated movement changes; impaired eccentric motor control); Decreased limb coordination resulting in step asymmetry;

- Fatigue and its associated influence on physical performance may lead to falls; Balance deficits (increased sway in stance and during functional tasks of daily living, delayed responses to perturbations, difficulty with tandem standing and walking) [16,51,52]; Gait impairments (decreased speed; stride length; stride width, increased variability in gait parameters) [53,54];

- Cognitive and behavioral issues including not recognizing their own disabilities and doing unsafe behaviors due to impulsiveness, attentional deficits and problems with dual tasking $[44,55,56]$; Deficits in spatial perception causing people with HD to run into walls or tables [57]; Visual disturbances such as difficulty with saccadic eye movements and smooth pursuit can impact balance and walking [58].

\section{General aims Treatment options}

- Improve mobility status (increase independence; increase speed; increase distance walked)

- Reduce risk of falls or actual falls ${ }^{\ddagger}$

- Maintain independent mobility including transfers and walking for as long as possible

- Reduce fear of falling which in itself may cause inactivity
- Impairment exercises: strengthening; general conditioning; endurance; range of motion activity to counteract effects of dystonia; coordination exercises; teach strategies to help people with HD identify when fatigue would increase their risk of falls [58,59]

- Balance training to practice the maintenance of postural control in a variety of tasks and environments

- Train patients to step in response to perturbations in all directions with speed and accuracy

- Practice activities that require automatic responses (e.g., throwing ball) to elicit postural responses and train faster movements

- Progress activities from wide to narrow BOS, static to dynamic activities, lowto-high COG, increasing degrees of freedom

- Task-specific practice of functional activities such as transfers, reaching high and low, stair climbing, etc. to train balance control during ADL

- Task-specific training to address walking tasks, ideally in specific environments (e.g. outdoor; obstacles); external cueing [16,59,60]

- Use metronome $[55,61,62]$, lines on floor to promote step initiation, bigger steps, faster speed, and gait symmetry

- Teach strategies as to how to get up from floor if they fall

- Teach safety awareness and adaptation of environment (reduce clutter, slippery surfaces, loose rugs, poor lighting, sharp or breakable objects), furniture

- Provision of assistive devices (4-wheeled walker with brakes) when appropriate; if patient is unsafe with assistive devices, human support such as holding the person's arm may be helpful [63]

- Family/carer education for guarding and/or assistance during ambulation

- Wheelchair prescription (long distance mobility)

- Shoe/orthotic evaluation (shoes with ankle support such as high top shoes or boots; heel wedge and/or lateral wedge for ankle dystonia in inversion/ eversion direction; ankle foot orthosis for ankle dystonia in dorsiflexion/ plantar flexion direction; custom made shoe inlay for individuals with clawing of toes during walking)

- Prescribe protective gear - helmets, elbow/knee pads to be worn by person with HD at risk for falls

- Teach compensatory strategies for cognitive impairments and inability to dual task

- Teach patients to focus their attention on maintaining balance before doing a task that challenges their balance [64]

- Practice two activities at same time under various practice and context conditions in early stages [55,56]

\section{Outcome measures:}

Function Assessment section of the UHDRS: which consists of the Functional Capacity Scale, the Independence Scale, and a checklist of common daily tasks for assessment of basic ADLs and IADLs. The total score on the Functional Capacity Scale is reported as the total functional capacity (TFC) score. The Independence Scale is rated from 0 to 100. Higher scores indicate better functioning [65].

HD-ADL: an informant rated instrument designed to follow disease progress. An ADL total score is calculated by summing all of the values for the five domains of Personal Care, Home Care, Work and Money, Social Relationships, and Communication. Validity and reliability has been demonstrated for subjects with HD on the seventeen items of adaptive functioning. Scores range from 0 (independent) to 24 (maximum disability) [66].

SF 36: see B; 10-m walk: see B; TUG: see B; 6MWT: see A.

ABC scale: measures of confidence performing various daily skills; participant rating scale [67].

Berg Balance Scale: Measures balance on 14 tasks; cutoff for falls risk in HD is a score of 40 [68,69].

Tinetti Mobility and Gait Test: Measures basic balance and walking ability [70,71]; Gait spatial and temporal measures: using GaitRite [72].

Falls history; UHDRS motor section

Interdisciplinary:

Nursing, occupational therapist, neuropsychologist, neurologist

tsee Management of chorea.

¥See Policy on falls and mobility, EHDN Physiotherapy Working Group. 
D. Secondary and adaptive changes and deconditioning.

Description: musculoskeletal and/or respiratory changes resulting in physical deconditioning, and subsequent decreased participation in daily living activities, or social work environments; stage: early-mid.

Signs and symptoms/key issues and

potential issues

Participation:

- Decreased level of physical fitness

- Decreased participation in ADLs, social or work environments

\section{Activities:}

- Decreased daily walking and physical activity levels in people with HD compared with healthy individuals [24]

- Daily walking levels significantly more reduced in people with HD who were recurrent fallers compared to those who were non-fallers [26]

\section{Impairments:}

- Musculoskeletal changes - loss of ROM, loss of strength due to inactivity [50]

- Respiratory changes - reduced endurance

- Cognitive problems such as memory deficits, loss of initiative or insight into problems [43]

- Psychological issues such as depression, apathy, anxiety

- Weight loss due to multiple factors may contribute to weakness, fatigue [73]

- Pain caused by dystonia, muscle imbalances, trauma from falls or hitting objects, immobility [74]

- Balance and gait impairments resulting in frequent falls (see C)

General aims

- Prevent further physical, cognitive, and psychological deterioration

- Improve strength, balance strategies and stamina

- Motivate patients to regain some control of their lives by adopting a healthy lifestyle

- Increase patient and caregiver awareness of benefits of regular exercise and detrimental effects of inactivity

- Weight control

- Pain management
Treatment options

- Educate patient and caregivers on benefits of exercise for people with HD and negative consequences of inactivity

- Initiate maintenance program to prevent secondary adaptive changes and deconditioning [75,76]

- Encourage person with HD to start/restart exercise program and provide exercise log or diary to record progress

- Consider formal or informal programs: individualized or group exercise programs in community/hospital settings [60]; exercise video or written instructions with pictures to increase home exercise compliance

- Assess for appropriate walking aids, devices (e.g., assistive, adaptive, orthotic)

- Treat balance and gait impairments, fear of falling that may underlie activity limitations [77]

- Educate caregivers on strategies to motivate (incorporate exercise into daily routine, positive reinforcement, participate in exercise with the patient, select physical activities that the patient enjoys) and assist their carers (i.e., cueing, guarding, use of gait belt) with exercise programs [78]

- Encourage patients to seek out enriched environments that are physically and cognitively stimulating and promote social interactions

- Teach breathing exercises to maintain full respiratory function

- Educate patient on importance of proper nutrition and maintenance of adequate weight

- Manage pain appropriately with modalities [79], ROM exercises, proper positioning, protection from injuries, medications, for example

\section{Outcome measures:}

SF-36: see A; Berg Balance Scale: see C; 6MWT: see A.

Impairment measures: aerobic capacity during functional activities, or during standardized exercise test (early stages); cardiovascular and pulmonary signs and symptoms in response to exercise or increased activity; Weight measurements; Mini Mental State Examination (MMSE) [80]; manual muscle testing or hand held dynamometry for muscle strength; Pain numerical rating scale, Pain Visual Analog scale (VAS), Wong-Baker FACES pain rating scale [74]; UHDRS Behavioral assessment section [65]; Goniometry, end feel assessment, and multisegment flexibility tests for ROM assessment; Respiratory rate, rhythm, and pattern, auscultation of breath sounds, cough effectiveness testing, vital capacity (VC) testing in supine and upright positions or forced vital capacity (FVC) testing for respiratory assessment.

Interdisciplinary:

Nutritionist, occupational therapist, psychologist, personal trainers. 
E. Impaired postural control and alignment in sitting.

Description: improper alignment due to adaptive changes, involuntary movement, muscle weakness and incoordination resulting in limitations in functional activities in sitting; stage: mid-late.
Signs and symptoms/ key issues and potential
General aims
Treatment options issues
Participation:
- Increased caregiver burden
- Withdrawal from society
Activities:
- Difficulty with ADLs, including washing, dressing
- Difficulty with feeding and swallowing
- Inability to stand or sit independently
Impairments:
- Inappropriate musculoskeletal alignment
- Decreased range of movement (active and passive)
- Soft-tissue adaptive changes
- Altered base of support leading to:
- Changes in dystonia/chorea
- Poor balance
- In-coordinated movement
- Pressure areas due to shearing
- Potential for falls and soft tissue damage
- Risk of aspiration and respiratory complications

\section{Outcome measures [29]:}

Carergiver Burden Scale: used to assess burden of care among caregivers. It is a 29-item scale designed to measure feelings of burden experienced by caregivers of elderly persons with senile dementia [83].

Goal Attainment Scale: decreased dependence in ADL, time tolerated in chair.

Interdisciplinary:

Nursing, speech language therapy for swallow assessment, respiratory physiotherapist, occupational therapist, liaise with specialist occupational therapist re: seating and hoist equipment

\section{F. Respiratory dysfunction.}

Description: impaired respiratory function and capacity; limited endurance; impaired airway clearance resulting in restrictions in functional activities and risk for infection; stage: mid-late.

$\begin{array}{ll}\text { Signs and symptoms/ key issues and potential issues } & \text { General aims } \\ \text { Participation: } & \text { - Optimize respiratory function for } \\ \text { - Restrictions in social activities e.g., shopping, family activities } & \text { functional activities } \\ \text { - Restrictions in exercise activities } & \text { - Optimize cardiorespiratory } \\ \text { Activities: } & \text { function } \\ \text { - Decreased exercise tolerance; limited ability to perform } & \text { - Maintain PCF } \geq 270 \mathrm{l} / \mathrm{min} \text { when } \\ \text { ADLs, ambulation } & \text { well; PCF } \geq 160 \mathrm{l} / \mathrm{min} \text { when } \\ \text { Impairments: } & \text { unwell with cold/respiratory } \\ \text { - Breathlessness on exertion or at rest } & \text { infection }[84] \\ \text { - Decreased exercise capacity } & \text { - Optimize secretion clearance }\end{array}$

- Ineffective cough

- Retained secretions

- Increased work of breathing

- Cyanosis

- Decreased oxygen saturation

- Dystonia of trunk muscles

Outcome measures:

Functional Assessment section of UHDRS (see C).

Borg breathlessness/MRC scale breathlessness scale: (Australian Lung Foundation).

6MWT (see A): if appropriate.

Peak cough flow [84].

Auscultation/observation/saturation monitor

Forced vital capacity

Interdisciplinary:

Speech and language therapy [89], nursing, respiratory therapist, pulmonologist.
Treatment options

- Functional exercise and ADL training

- Positioning to manage breathlessness [85]

- Breathing exercises; maximal insufflation/ exsufflation; glossopharyngeal breathing $[84,86]$

- Airway clearance techniques [87]

- Postural management [88]

- Relaxation

- Consultation for appropriate walking aids e.g., wheeled walker/rollator

- Cardiovascular exercise training [86] 


\section{G. End stage care.}

Description: active and passive range of motion limitations and poor active movement control resulting in inability to ambulate; dependent for most ADLs; difficulty maintaining upright sitting position; stage: late.

\author{
Signs and symptoms/key issues \\ and potential issues \\ Participation: \\ - Complete dependence in \\ functional skills; social isolation \\ Activities: \\ - Unable to ambulate; dependent for \\ most ADLs; difficulty maintaining \\ upright sitting position \\ Impairments: \\ - Limited volitional control of limbs \\ and trunk chorea and/or rigidity \\ - Limitations in passive range of \\ motion \\ - Risk for aspiration/respiratory \\ infection \\ - Risk for pressure sores; pain due \\ to positioning or pressure sores or \\ contractures \\ - Difficulties or inability to \\ communicate \\ - Depressed mood or depression \\ - Pain
}

\section{General aims \\ Treatment options}

- Minimize risk of aspiration/respiratory Positioning: infection

- Minimize risk of bed sores

- Promote ability to maintain upright sitting position

- Promote optimal comfort in bed

- Maintain/increase range of motion

- Maintain existing ADLs

- Support and brief nursing staff or caregivers

- If necessary: provide support to organize external help (homenursing services) or relocation to a specialized institution

- In bed - utilize pressure relieving mattresses and cushions for optimal positioning; utilize positioning schedule in bed to promote position change (side-lying and supine).

- Upright - consultation for appropriate supportive chair: key features include padded supports (to prevent injury secondary to involuntary movements), tilt in space to maintain appropriate hip angle and allow for change in position for pressure relief; adequate trunk and head supports; adequate padded foot supports.

- Close cooperation with nursing staff / caregiver to improve transfers, eating position, communication, washing and dressing and to preserve whatever independence the patient has in terms of ADL's. Advise as to signs of aspiration [75].

Range of motion: create range of motion exercise plan to be performed daily by nursing staff, aides, or family members. Regular physiotherapy sessions advisable [75].

Active movement: if able, encourage upright standing with support (consider use of standing table); sitting on edge of bed (with support); active exercises in bed to prevent muscle wasting and prevent breakdown; work with existing capabilities to maintain current ADLs [75].

Respiratory: see Respiratory TC.

\section{Outcome measures}

Braden Risk Assessment Scale: grading tool for risk of pressure ulcers [90].

National Pressure Ulcer Advisory Panel (NPUAP): pressure ulcer staging [101]; respiratory function assessment; ability to sit upright in adaptive chair for measured period of time; LE/UE range of motion assessment using goniometer; pain assessment with FACES pain scale [74]; Caregiver Burden Scale [83] if patient remains at home.

Goal Attainment Scale (GAS) [29]: see A.

Interdisciplinary:

Nursing, respiratory therapy, speech \& language therapy, clergy for spiritual support, psychologists for grief counselling, occupational therapist for seating, social workers for living wills, power of attorney. 


\section{References}

1 Fritz JM, Brennan GP. Preliminary examination of a proposed treatment-based classification system for patients receiving physical therapy interventions for neck pain. Phys. Ther. 87(5), 513-524 (2007).

2 Scheets PL, Sahrmann SA, Norton BJ. Use of movement system diagnoses in the management of patients with neuromuscular conditions: a multiple-patient case report. Phys. Ther. 87(6), 654-669, (2007).

3 Bello-Haas VD. A framework for rehabilitation of neurodegenerative diseases: planning care and maximizing quality of life. Neurol. Rep. 26(3), 115-129 (2002).

4 Ramaswamy B, Ashburn A, Durrant K et al. Quick reference cards (UK) and guidance notes for physiotherapists working with people with PD. Parkinson Disease Society (2009).

5 Royal Dutch Society for Physical Therapy. KGNF Guidelines for Physical Therapy in Patients with Parkinson's Disease. Dutch J. Physiother. 114(Suppl. 3), (2004).

6 Vaccarino AL, Sills T, Anderson KE et al. Assessment of day-to-day functioning in prodromal and early Huntington disease. PLoS Curr. 3, RRN1262 (2011).

7 Morton AJ, Wood NI, Hastings MH, Hurelbrink C, Barker RA, Maywood ES. Disintegration of the sleep-wake cycle and circadian timing in Huntington's disease. J. Neurosci. 25(1), 157-163 (2005).

8 van Dellen A, Cordery PM, Spires TL, Blakemore C, Hannan AJ. Wheel running from a juvenile age delays onset of specific motor deficits but does not alter protein aggregate density in a mouse model of Huntington's disease. BMC Neurosci. 9, 34 (2008).

9 Dobrossy MD, Dunnett SB. Training specificity, graft development and graftmediated functional recovery in a rodent model of Huntington's disease. Neuroscience 132(3), 543-552 (2005).

10 Trembath MK, Horton ZA, Tippett L et al. A retrospective study of the impact of lifestyle on age at onset of Huntington disease. Mov. Disord. 25(10), 1444-1450 (2010).

11 Scarmeas, N, Levy G, Tang MX, Manly J, Stern Y. Influence of leisure activity on the incidence of Alzheimer's disease. Neurology 57(12), 2236-2242 (2001).

12 Thacker, EL, Chen H, Patel AV et al. Recreational physical activity and risk of Parkinson's disease. Mov. Disord. 23(1), 69-74 (2008)
13 Quinn L, Busse M, Khalil $\mathrm{H}$ et al. Client and therapist views on exercise programmes for early-mid stage Parkinson's disease and Huntington's disease. Disabil. Rehab. 32(11), 917-928 (2010).

14 van Nimwegen M, Speelman AD, Smulders K et al. Design and baseline characteristics of the ParkFit study, a randomized controlled trial evaluating the effectiveness of a multifaceted behavioral program to increase physical activity in Parkinson patients. BMC Neurol. 10, 70 (2010).

15 Goodwin V, Richards SH, Taylor RS, Taylor $\mathrm{AH}$, Campbell JL. The effectiveness of exercise interventions for people with Parkinson's disease: a systematic review and meta-analysis. Mov. Disord. 23(5), 631-640 (2008).

16 Quinn L, Rao A. Physical therapy for people with Huntington disease: current perspectives and case report. Neurol. Rep. 26(3), 145-153 (2002).

17 Meaney, A, Busse M, Dawes H, Rosser A. Response to a structured exercise programme for Huntington's Disease; a single case study. Br. Assoc. Sports Exer. Med. J Sports Sci. (2008).

18 Busse M, Khalil H, Quinn L, Rosser A. Physical therapy intervention for people with Huntington disease. Phys. Ther. 88(7), 820-831 (2008).

19 American College of Sports Medicine. Guidelines for Exercise Testing and Prescription. $8^{\text {th }}$ Edition. Lippincott Williams \& Wilkins (PA, USA) (2009)

20 Kloos A, Kostyk S, Kegelmeyer D. The effect of video game-based exercise on dynamic balance, mobility and UHDRS neuropsychiatric test scores in individuals with Huntington's Disease. J. Am. Soc. Experiment. Neurother. 8(1), 139 (2011).

21 Thomas S, Reading J, Shephard RJ. Revision of the Physical Activity Readiness Questionnaire (PAR-Q). Can. J. Sport Sci. 17(4), 338-345 (1992).

22 Craig C, Marshall AL, Sjostrom M et al. International physical activity questionnaire: 12-country reliability and validity. Med. Sci. Sports Exerc. 35(8), 1381-1395 (2003).

23 van Vugt JP, Siesling S, Piet KK et al. Quantitative assessment of daytime motor activity provides a responsive measure of functional decline in patients with Huntington's disease. Mov. Disord. 16(3), 481-488 (2001).

24 van Vugt JP, Piet KK, Vink LJ et al. Objective assessment of motor slowness in Huntington's disease: clinical correlates and 2-year followup. Mov. Disord. 19(3), 285-297 (2004).
25 Busse ME, van Deursen RW, Wiles CM. Activity indices for measuring mobility in neurologically impaired patients. J. Neurol. Neurosurg. Psychiatry 74(10), 1459-1459 (2003).

26 Busse ME, Wiles CM, Rosser AE. Mobility and falls in people with Huntington's disease. J. Neurol. Neurosurg. Psychiatry 80(1), 88-90 (2009).

27 Noble BJ, Robertson RJ. Perceived Exertion. Human Kinetics, IL, USA (1996).

28 Day ML, McGuigan MR, Brice G, Foster C. Monitoring exercise intensity during resistance training using the session RPE scale. J. Strength Condition. Res. 8(2), 53-358 (2004).

29 Turner-Stokes L. Goal attainment scaling (GAS) in rehabilitation: a practical guide. Clin. Rehabil. 23(4), 362-370 (2009).

30 Enright PL. The six-minute walk test. Respir. Care 48(8), 783-785 (2003)

31 West C, Bowen A, Hesketh A, Vall A. Interventions for motor apraxia following stroke. Cochrane Database Syst. Rev. 2008(1), CD004132 (2008).

32 Donkervoort M, Dekker J, Stehmann-Saris FC. Efficacy of strategy training in left hemisphere stroke patients with apraxia: a randomized clinical trial. Neuropsych. Rehab. 11(5), 549-566 (2011).

33 Melton AK, Bourgeois MS. Training compensatory memory strategies via telephone for persons with TBI. Aphasiology 19, 353-364 (2005)

34 Leng TR, Woodward MJ, Stokes MJ, Swan AV, Vareing LA, Baker R. Effects of multisensory stimulation in people with Huntington's disease: a randomized controlled pilot study. Clin. Rehabil. 17(1), 30-41 (2003).

35 Ho AK, Robbins AO, Walters SJ, Kaptoge S, Sahakian BJ, Barker RA. Health-related quality of life in Huntington's disease: a comparison of two generic instruments, SF-36 and SIP. Mov. Disord. 19(11), 1341-1348 (2004).

36 Watson MJ. Refining the ten-metre walking test for use with neurologically impaired people. Physiotherapy 88(7), 386-397 (2002).

37 Podsiadlo D, Richardson S. The timed "Up \& Go": a test of basic functional mobility for frail elderly persons. J. Am. Geriatr. Soc. 39(2), 142-148 (1991).

38 Rao AK, Muratori L, Louis ED, Moskowitz CB, Marder KS. Clinical measurement of mobility and balance impairments in Huntington's disease: validity and responsiveness. Gait Posture 29(3), 433-436 (2009). 
39 Whitney S, Marchetti GF, Morris LO, Sparto PJ. The reliability and validity of the four square step test for people with balance deficits secondary to vestibular disorder. Arch. Phys. Med. Rehabil. 88, 99 (2008).

40 VanHeugten CM, Dekker J, Deelman BG, Stehmann-Saris JC, Kinebanian A. A diagnostic test for apraxia in stroke patient: internal consistency and diagnostic value. Clin. Neuropsychol. 13, 182 (1999).

41 Whitney SL, Wrisley DM, Marchetti GF, Gee $\mathrm{MA}$ et al. Clinical measurement of sit-tostand performance in people with balance disorders: validity of data for the five-timessit-to-stand test. Phys. Ther. 85(10), 1034-1045 (2005).

42 Lasker AG, Zee DS. Ocular motor abnormalities in Huntington's disease. Vision Res. 37(24), 3639-3645 (1997).

43 Lemiere J, Decruyenaere M, Evers-Kiebooms G, Vandenbusche E, Dom R. Cognitive changes in patients with Huntington's disease (HD) and asymptomatic carriers of the HD mutation - a longitudinal follow-up study. J. Neurol. 251(8), 935-942 (2004).

44 Grimbergen Y, Knol MJ, Bloem BR, Kremer BP, Roos RA, Munneke M. Falls and gait disturbances in Huntington's disease. Mov. Disord. 23(7), 970-976 (2008).

45 Aubeeluck A, Wilson E. Huntington's disease. Part 1: essential background and management. Br. J. Nurs. 17(3), 146-151 (2008).

46 Louis ED, Lee P, Quinn L, Marder K. Dystonia in Huntington's disease: prevalence and clinical characteristics. Mov. Disord. 4(1), 95-101 (1999).

47 Penney, JB, Vonsattel JP, MacDonald ME, Gusella JF, Myers RH. CAG repeat number governs the development rate of pathology in Huntington's disease. Ann. Neurol. 41(5), 689-692 (1997).

48 Rosenblatt A, Abbott MH, Gourley LM et al. Predictors of neuropathological severity in 100 patients with Huntington's disease. Ann. Neurol. 54(4), 488-493 (2003).

49 Vonsattel JP, Keller C, Cortes Ramirez EP. Huntington's disease - neuropathology. Handbook Clin. Neurol. 100, 83-100 (2011).

50 Busse M, Hughes G, Wiles CM, Rosser AE. Use of hand-held dynamometry in the evaluation of lower limb muscle strength in people with Huntington's disease. J. Neurol. 255(10), 1534-1540 (2008).

51 Tian J, Herdman SJ, Zee DS, Folstein SE. Postural stability in patients with Huntington's disease. Neurology 42(6), 1232-1238 (1992).
52 Panzera R, Salomonczyk D, Pirogovosky E et al. Postural deficits in Huntington's disease when performing motor skills involved in daily living. Gait Posture 33(3), 457-461 (2011).

53 Rao A, Muratori L, Louis L, Moskowtiz C, Marder K. Spectrum of gait impairments in presymptomatic and symptomatic Huntington's disease. Mov. Disord. 23(8), 1100-1107 (2008).

54 Hausdorff, JM. Gait variability and basal ganglia disorders: stride-to-stride variations of gait cycle timing in Parkinson's disease and Huntington's disease. Mov. Disord. 13(3), 428-437 (1998).

55 Delval A, Krystkowiak P, Delliaux M et al. Effect of external cueing on gait in Huntington's disease. Mov. Disord. 23(10), 1446-1452 (2008).

56 Delval A, Krystkowiak P, Delliaux M et al. Role of attentional resources on gait performance in Huntington's disease. Mov. Disord. 23(5), 684-689 (2008).

57 O'Donnell BF. Visual perception in prediagnostic and early stage Huntington's disease. J. Int. Neuropsychol. Soc. 14(3), 446-453 (2008).

58 Hicks, SL, Robert MP, Golding CV, Tabrizi SJ, Kennard C. Oculomotor deficits indicate the progression of Huntington's disease. Prog. Brain Res. 171, 555-558 (2008).

59 Peacock IW. A physical therapy program for Huntington's disease patients. Clin. Manag. Phys. Ther. 7(1), 22-23 (1987).

60 Zinzi P, Salmaso D, De Grandis R et al. Effects of an intensive rehabilitation programme on patients with Huntington's disease: a pilot study. Clin. Rehabil. 21(7), 603-613 (2007).

61 Thaut MH, Miltner R, Lange HW, Hurt CP, Hoemberg V. Velocity modulation and rhythmic synchronization of gait in Huntington's disease. Mov. Disord. 14(5), 808-819 (1999).

62 Churchyard AJ, Morris ME, Georgiou N, Chiu E, Cooper R, Iansek R. Gait dysfunction in Huntington's disease: parkinsonism and a disorder of timing. Implications for movement rehabilitation. Adv. Neurol. 87, 375-385 (2001).

63 Kloos A, Kegelmeyer D, Kostyk S. The effects of assistive devices on gait measures in Huntington's disease. Neurotherapeutics 6(1), 209-210 (2009).

64 Bilney B, Morris ME, Denisenko S. Physiotherapy for people with movement disorders arising from basal ganglia dysfunction. NZ J. Physiother. 31(2), 94-100 (2003).
65 Huntington Study Group. Unified Huntington's DISEASE Rating Scale: reliability and consistency. Mov. Disord. 11, 136-142 (1996).

66 Bylsma FW, Rothlind J, Hall MR, Folstein SE, Brandt J. Assessment of adaptive functioning in Huntington's disease. Mov. Disord. 8(2), 183-190 (1993).

67 Powell LE, Myers AM. The Activities-specific Balance Confidence (ABC) Scale. J. Gerontol. A Biol. Sci. Med. Sci. 50A(1), M28-M34 (1995).

68 Rao A, Muratori L, Louis ED, Moskowitz $\mathrm{CB}$, Marder KS. Clinical measurement of mobility and balance impairments in Huntington's disease: validity and responsiveness. Gait Posture 29(3), 433-436 (2009).

69 Berg KO, Wood-Dauphinee SL, Williams JI, Maki B. Measuring balance in the elderly: validation of an instrument. Can. J. Public Health 83(Suppl. 2), S7-S11 (1992).

70 Tinetti ME. Performance-oriented assessment of mobility problems in elderly patients. J. Am. Geriatr. Soc. 34(2), 119-126 (1986).

71 Kloos AD, Kegelmeyer DA, Young GS, Kostyk SK. Fall risk assessment using the Tinetti mobility test in individuals with Huntington's disease. Mov. Disord. 25(16), 2838-2844 (2010).

72 Rao AK, Quinn L, Marder KS. Reliability of spatiotemporal gait outcome measures in Huntington's disease Mov. Disord. 20 (8), 1033-1037 (2005).

73 Aziz NA. Weight loss in Huntington disease increases with higher CAG repeat number. Neurology 71(19), 1506-1513 (2008).

74 Wong D. Pain in children: comparison of assessment scales. Pediat. Nurs. 14, 9-17 (1998).

75 Jackson J. Specific treatment techniques. In: Physical Management for Neurological Conditions (3rd Edition). Stokes M, Stokes E (Eds). Churchill Livingstone Elsevier, 243-266 (2011).

76 Haas BA. Physical activity and exercise in neurological rehabilitation. In: Physical Management for Neurological Conditions (3rd Edition). Stokes M, Stokes E (Eds). Churchill Livingstone Elsevier, 349-365 (2011).

77 Kunkel D, Stack E. Falls and their management. In: Physical Management for Neurological Conditions (3rd Edition). Stokes M, Stokes E (Eds). Churchill Livingstone Elsevier, 383-397 (2011).

78 Jones F. Self management. In: Physical Management for Neurological Conditions (3rd Edition). Stokes M, Stokes E (Eds). Churchill Livingstone Elsevier, 367-387 (2011). 
79 Watson P. Pain management in neurological rehabilitation. In: Physical Management for Neurological Conditions (3rd Edition). Stokes M, Stokes E (Eds). Churchill Livingstone Elsevier, 331-338 (2011).

80 Folstein MF, Robins LN, Helzer JE. The Mini-Mental State Examination. Arch. Gen. Psychiatry 40(7), 812 (1983).

81 Kilbride CC. Physical management of altered tone and movement. In Physical Management for Neurological Conditions (3rd Edition). Stokes M, Stokes E (Eds). Churchill Livingstone Elsevier, 289-318 (2011).

Bruton A. Respiratory management in neurological rehabilitation. In: Physical Management for Neurological Conditions (3rd Edition). Stokes M, Stokes E (Eds). Churchill Livingstone Elsevier, 319-330 (2011).

83 Zarit SH, Reever KE, Bach-Peterson J. Relatives of the impaired elderly: correlates of feelings of burden. Gerontologist 20, 649-655 (1980).

84 Bott J. Guidelines for the physiotherapy management of the adult, medical, spontaneously breathing patient. Thorax 64(Suppl. 1), i1-i52 (2009).

85 Moxham J. Breathlessness, fatigue and the respiratory muscles. Clin. Med. J. Royal Coll. Phys. 9(5), 448-452 (2009).

86 Jones U, Enright S, Busse M. Management of respiratory problems in people with neurodegenerative conditions: a narrative review. Physiotherapy doi:10.1016/j. physio.2011.03.002 (2011) (Epub ahead of print).

87 McCool FD, Rosen MJ. Nonpharmacologic airway clearance therapies: ACCP evidencebased clinical practice guidelines. Chest 129(1 Suppl.), 250S-259S (2006).
88 Dysfunctional breathing. In: Physiotherapy for Respiratory and Cardiac Problems. Prasad PA (Ed.). Churchill Livingstone, Oxford, UK (2008).

89 Yorkston KM, Millar RM, Klasner ER. Huntington's Disease. In: Management of Speech and Swallowing in Degenerative Disease. KM, Yorkston KM, RM Millar \& Stroud EA (Eds). PRO-ED, TX, USA 139-154 (2004).

90 Braden BJ, Bergstrom N. Predictive validity of the Braden scale for pressure sore risk in a nursing home. Res. Nurs. Health 17, 459-470 (1994).

\section{- Website}

101 European Pressure Ulcer Advisory panel (EPUAP and national Pressure Ulcer Advisory Panel (NPUAP). Pressure Ulcer Treatment: Quick Reference Guide. www.npuap.org 